\title{
Percepción del dolor según el modo de punción de la fístula arteriovenosa en pacientes sometidos a hemodiálisis. Revisión Sistemática
}

\author{
Manuel Pabón Carrasco ${ }^{1}$, Eva del Rocío Martínez Alfonso², Samuel Vilar Palomo³, María Inmaculada López \\ Leiva $^{4}$ \\ ${ }^{1}$ Departamento Enfermería. Centro Universitario de enfermería Cruz Roja, adscrita a la Universidad de Sevilla. \\ España \\ 2 Graduada en Enfermería \\ ${ }^{3}$ Hospital Universitario Virgen del Rocío. Unidad del Dolor. España \\ ${ }^{4}$ Departamento de Enfermería. Facultad de Enfermería y Podología. Universidad de Málaga. España
}

\section{Resumen}

Objetivo: Valorar la posible asociación entre la forma de punción de la fístula arteriovenosa y el dolor que siente el paciente por la inserción de la aguja.

Metodología: Se ha seguido la declaración PRISMA para revisiones sistemáticas. Se han consultado 6 bases de datos; Medline, Scopus, Cuiden, CINAHL, SciELO, y Cochrane PLUS. Además, se realizó una búsqueda secundaría manual para detectar literatura gris no encontrada en las bases de datos o mediantes las estrategias definidas. Criterios de inclusión; estudios de nivel de evidencia 1 en la escala Scottish Intercollegiate Guidelines Network, publicados en los últimos 10 años, idiomas español/inglés, y temática relacionada con las técnicas utilizadas en hemodiálisis para el abordaje de la fistula. Los artículos se evaluaron de forma crítica para detectar cualquier riesgo de sesgo mediante el instrumento CASPe y el manual Cochrane para Revisiones Sistemáticas de Intervenciones.

Resultados: Se identificaron un total de 150 resultados, seleccionándose finalmente 9 estudios. Los resultados han sido muy heterogéneas, encontrándose resultados para el dolor, tasas de infección, número de intentos de canalización, flujo sanguíneo, presión venosa, tiempo



de hemostasia, seguridad, dilataciones aneurismáticas, estética del brazo portador de la fístula y calidad de vida del paciente.

Conclusiones: La evidencia no respalda el uso preferencial de la técnica del ojal sobre la canalización tradicional. Por tanto, se necesita mejorar el rigor metodológico y un mayor tamaño muestral para dilucidar que técnica da mejores prestaciones respecto al dolor, permitiendo por tanto mejorar la calidad de vida de los pacientes de hemodiálisis.

PALABRAS CLAVE: fístula arteriovenosa; hemodiálisis; percepción del dolor; dolor.

\section{Pain perception according to manner of arteriovenous fistula cannulation in patients undergoing hemodialysis. Systematic review}

\section{Abstract}

Aims: To assess the possible association between the manner of arteriovenous fistula cannulation and the patient pain related to the insertion of the needle for the hemodialysis session.

Methodology: The PRISMA statement for systematic reviews has been followed. Six databases have been consulted: Medline, Scopus, Cuiden, CINAHL, SciELO, and Cochrane PLUS. In addition, a secondary manual search was performed to detect grey literature not found 
in databases or through defined strategies. Inclusion criteria: studies of level of evidence 1 on the Scottish Intercollegiate Guidelines Network scale, published in the last 10 years, Spanish/English languages, and topics related to the techniques used to canalize the hemodialysis fistula. The articles were critically evaluated to detect any risk of bias using the CASPe instrument and the Cochrane Handbook for Systematic Reviews of Interventions.

Results: A total of 150 results were identified, 9 of which were finally selected. The results have been very heterogeneous, finding results for pain, infection rates number of attempts of channalization, blood flow, venous pressure, time of hemostasis, safety, aneurysmal dilations, esthetics of the fistula-carrying arm and quality of life of the patient.

Conclusions: The evidence does not support the preferential use of the buttonhole technique over traditional channeling. Therefore, it is necessary to improve the methodological quality and a larger sample size to elucidate which technique gives better benefits with respect to pain, thus allowing to improve the quality of life of hemodialysis patients.

KEYWORDS: arteriovenous fistula; hemodialysis; pain perception; pain.

\section{Introducción}

Se está produciendo en nuestra sociedad un incremento progresivo en la incidencia de la enfermedad renal crónica (ERC). En el estadio final de la ERC, el paciente requiere tratamiento renal sustitutivo precisando, por tanto, de diálisis (diálisis peritoneal o hemodiálisis) y/o de trasplante renal. Se estima que en 2012 , un $80,6 \%$ de los pacientes con ERC iniciaron un tratamiento con $\mathrm{HD}^{1}$, por tanto es fundamental un acceso vascular adecuado para llevar a cabo esta técnica. Los más comunes son los accesos arteriovenosos como la fístula arteriovenosa interna (FAV), o los injertos arteriovenosos ${ }^{2,3}$. EI acceso a la FAV se realiza a través de la bipunción periódica, normalmente tres veces por semana en función de las características del tratamiento de HD y el éxito de la canalización es fundamental para la realización de un tratamiento satisfactorio. Anualmente, un paciente puede recibir 312 punciones, que además de repercutir de forma negativa en el mantenimiento de la fístula arteriovenosa, también generan estados de ansiedad en el paciente ${ }^{4}$. Un estudio realizado por Moreno et al., de- mostró que un $46,6 \%$ de los pacientes hemodializados en un hospital de Alicante presentaban síntomas de ansiedad $^{5}$. En otra unidad de hemodiálisis donde se valoró la satisfacción de los usuarios, fue el control del dolor el ítem más destacado ${ }^{6}$. La punción es un factor muy importante para mantener una buena calidad en la diálisis y por el impacto que genera en el paciente ${ }^{7}$. Numerosos estudios, reflejan que según la técnica empleada, es posible reducir el dolor de la punción de la FAV, así como la supervivencia del acceso vascular, la tasa de infección y otras complicaciones asociadas a la canalización². Existen tres formas de abordaje, por un lado está la técnica de punción en escalera de cuerda conocida en el ámbito anglosajón como "Rope Ladder". Esta consiste en distribuir las punciones de la fístula arteriovenosa a lo largo de toda su longitud. La técnica del área de punción se fundamenta en realizar las punciones siempre en un área concreta entre $2-3 \mathrm{~cm}$. Esta es la más utilizada porque reduce el número de punciones complicadas. Por último, la técnica de buttonhole o técnica del ojal, que se basa en realizar la punción siempre sobre el mismo punto, con el mismo ángulo y la misma profundidad en cada sesión de hemodiálisis, generando así la creación de un túnel subcutáneo que facilita las punciones. En España, las técnicas de punción convencionales, en área de punción y en escalera de cuerda, han sido las más empleadas ya que son sencillas de ejecutar por los profesionales formados en diálisis, pero son responsables de gran morbilidad a medio y largo plazo asociada al acceso vascular, ya que ocasionan pequeños traumatismos que debilitan la pared del vaso sanguíneo formando un aneurisma en la zona de punción de la FAV8,9. Este proceso fisiopatológico se denomina remodelado iatrogénico ${ }^{10}$. Por otro lado, la técnica del ojal es poco conocida y no hay tanta información sobre ella $a^{4,6}$.

Un estudio realizado por Kosa et al., en 2016, donde se valoró el grado de satisfacción de los pacientes hemodializados, muestra en sus resultados que uno de los ítems peor valorado es la canalización física de la fistula 0 injertos ${ }^{11}$. La necesidad de velar por la calidad de vida del paciente es una tarea fundamental de los profesionales de enfermería, así como mejorar la tolerancia del mismo frente a su situación, evitando respuestas vaso-vagales que compliquen el acceso a la $\mathrm{FAV}^{2,6}$. Ante las diferentes técnicas de elección que nos brinda la práctica clínica respecto a la forma de abordar la punción y el incremento en el número de pacientes que precisan de estos cuidados, se propone la presente revisión sistemática con el fin de conocer la percepción del dolor en los pacientes hemodializados en función de las técnicas aplicadas. 


\section{Metodología}

Se realizó una revisión sistemática siguiendo las indicaciones del Manual Cochrane ${ }^{12}$ y las recomendaciones del informe PRISMA ${ }^{13}$.

Para realizar esta revisión se ha seguido una serie de etapas: 1. Definición del tema. 2. Definición de la pregunta de investigación. 3. Especificación de los criterios de inclusión y exclusión. 4. Elaboración del plan de trabajo para realizar la búsqueda de la literatura por pares. 5. Selección y recuperación de los estudios que cumplen los criterios. 6. Valoración por pares de la calidad metodológica de los estudios. 7. Extracción de los datos. 8. Análisis y síntesis de los Resultados. 9. Formulación de las Conclusiones ${ }^{12,14}$.

\section{Criterios de inclusión y exclusión}

Se incluyeron aquellos estudios que cumplían las siguientes características:

- Estudios sobre técnicas de buttonhole/técnica del ojal o punción en escalera de cuerda o área de punción.

- Estudios con un nivel metodológico de 1 en la escala SIGN: Meta-análisis, revisiones sistemáticas de ensayos clínicos aleatorizados o ensayos clínicos aleatorizados.

- Estudios dirigidos y llevados a cabo en humanos.

- Estudios de los últimos 10 años.

- Estudios con suficiente calidad metodológica según el instrumento CASPe para ensayos clínicos y revisiones $^{15}$.

Se excluyeron aquellos estudios que no valoraban la dimensión dolor entre sus variables de estudio o cuya metodología era deficiente. Los estudios realizados en idiomas distintos el inglés y el español fueron excluidos.

\section{Fuentes de información y estrategia de búsqueda}

Se realizaron búsquedas iniciales en bases de datos electrónicas desde 6 de febrero de 2018 hasta el 30 de junio del 2018. Las fuentes consultadas para la elaboración de esta revisión fueron Medline, Scopus, Cuiden, CINAHL, SciELO, y Cochrane PLUS. También se realizó una búsqueda secundaría manual en las lista de referencias de los artículos seleccionados. Se utilizaron descriptores en castellano o inglés según la base de datos Decs/MesH: "Hemodiálisis"; "fistula arteriovenosa"; "dolor" y "percepción del dolor". Además de términos libres: "técnica del ojal"; "punción en escalera de cuerda", "área de punción", "técnica de canalización"y "métodos convencionales". Mediante el uso combinado de booleanos AND/OR/NOT, truncamientos y comillas. Para Medline, los términos de búsqueda incluyeron: (hemodialysis OR "arteriovenous fistula") AND (pain OR "Pain Perception") AND (Buttonhole OR "rope-ladder" OR puncture area OR cannulation technique OR "tradicional method") obteniendo sin filtros un total de 90 artículos potencialmente elegibles. Se utilizó una segunda estrategia, para acotar la búsqueda, eliminando el término hemodiálisis: ("arteriovenous fistula") AND (pain OR "Pain Perception") AND (Buttonhole OR "rope-ladder" OR puncture area OR cannulation technique OR "tradicional method"): 28 artículos potencialmente válidos.

Los resultados fueron comparados con el software Mendeley ${ }^{\circledast}$, y los duplicados fueron eliminados. Revisamos el título y el resumen de los estudios restantes para detectar los relevantes (MPC). Los textos íntegros de los estudios posiblemente adecuados fueron leídos por dos autores (MILL/ERMA) para valorar su elegibilidad según los criterios de inclusión. Examinamos los listados de referencias de los manuscritos elegidos y de artículos de revisión relevantes para buscar estudios potencialmente elegibles.

\section{Proceso de extracción de datos}

Una revisora (ERMA) extrajo los datos y consultó a un investigador de mayor categoría (SVP). De los artículos seleccionados se extrajeron la autoría, año y lugar de publicación, estudio de diseño, tamaño muestral, temática y nivel de evidencia según la escala SIGN. Los revisores (MILL/MPC) no fueron cegados por autor o institución en ninguna etapa del proceso de selección o de recopilación de datos.

\section{Riesgo de sesgo en los estudios individuales}

El riesgo de sesgo fue valorado con los instrumentos para la lectura crítica CASPe y el manual Cochrane Handbook for Systematic Reviews of Interventions. Dos autores (MILL/MPC) valoraron el riesgo de sesgo de forma independiente. La calificación de la calidad de los estudios se realizó según la especialización en el campo (dirigido por SVP) y se guió con una herramienta de valoración de sesgo. 


\section{Resultados}

\section{Participantes y estudios incluidos}

La Figura 1 detalla el flujograma PRISMA ${ }^{16}$. Este estudio se realizó usando 6 bases de datos, y tras eliminar los duplicados, el cribado se hizo con veintiuno estudios. Nueve de los veintiuno mencionados fueron seleccionados para la revisión sistemática. La Tabla 1 muestra las características de los estudios tras aplicar todos los criterios de elegibilidad.

La Figura 1 detalla el flujograma PRISMA Medline, Scopus, Cuiden, CINAHL, SciELO, y Cochrane PLUS.

\section{Descripción de los resultados}

De los nueve estudios analizados, seis son ensayos clínicos aleatorios con un aceptable nivel metodológico, dos son revisiones sistemáticas con sesgo de selección debido a la heterogeneidad de los resultados analizados. Finalmente la muestra de estudios está compuesta por un Meta-análisis con alto nivel metodológico.
En cuanto al idioma, los nueve estudios estaban publicados en inglés. Además, dos de estos estudios fueron desarrollados en Europa, uno en Oceanía, tres en América y tres en Asia.

Todos los estudios informaron de las características iniciales de los pacientes, incluidos la edad (media 60 años), el sexo (mayoritariamente hombres), la duración de la diálisis y las comorbilidades. En la mayoría de los estudios, no hubo una diferencia significativa de las características iniciales entre los grupos de experimentación y control. La mayoría de los ensayos controlados aleatorios carecían de cegamiento y ocultamiento de la asignación, además se encuentran diferencias sustanciales en la definición de la medida de resultado y los formatos de los datos. La duración del seguimiento varió de 3 a 18 meses con diferencias significativas, lo que llevó a la heterogeneidad de los resultados del estudio.

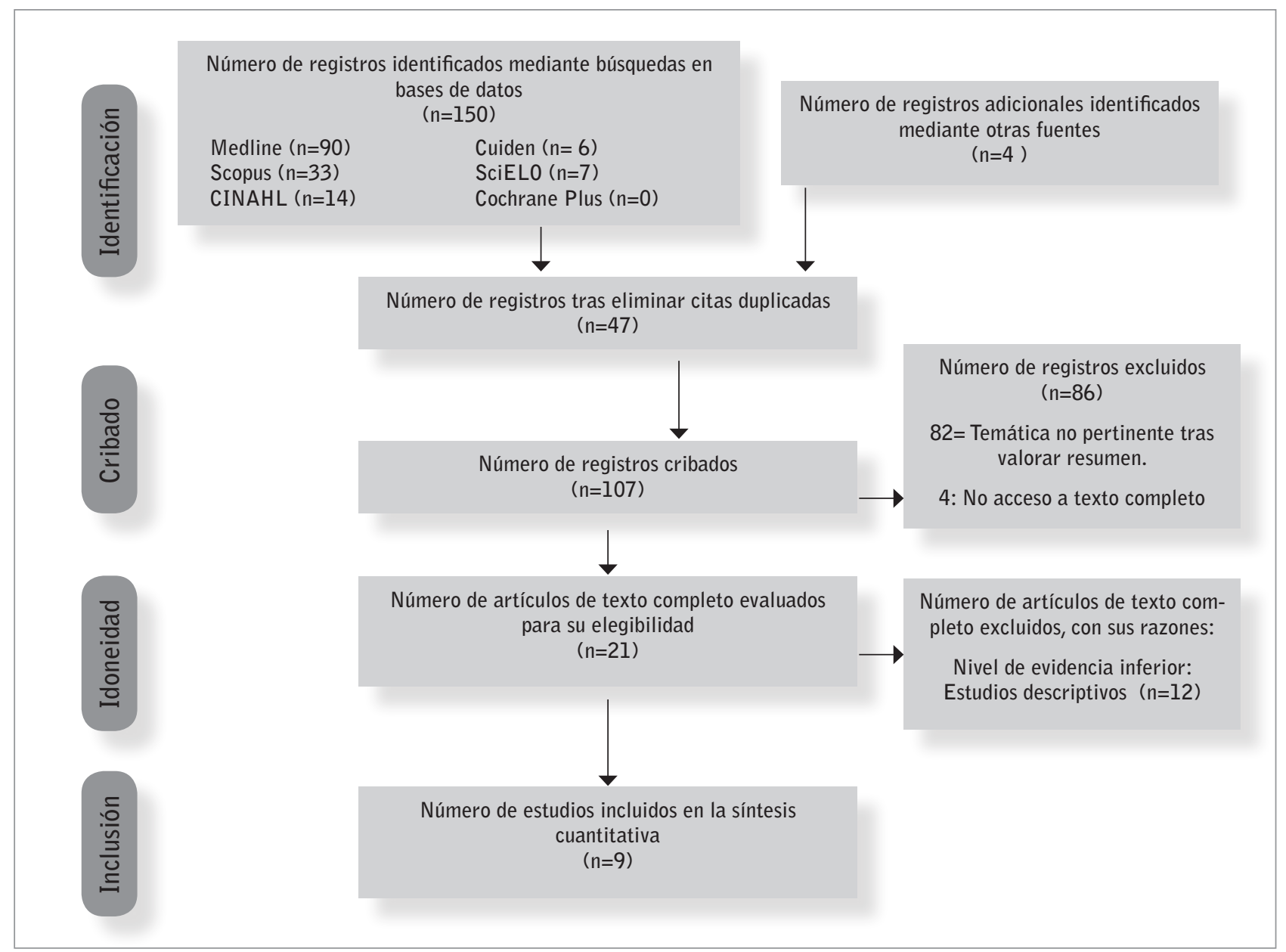

Figura 1. Flujograma PRISMA ${ }^{17}$. 
Tabla 1. Características de los estudios que se seleccionaron para llevar a cabo esta revisión.

\begin{tabular}{|c|c|c|c|c|c|}
\hline $\begin{array}{l}\text { Estudio } \\
\text { Nivel } \\
\text { Evidencia }\end{array}$ & $\begin{array}{l}\text { Número de } \\
\text { participantes } \\
\text { y país }\end{array}$ & Diseño & Seguimiento & Objetivos & Resultados \\
\hline $\begin{array}{l}\text { Struthers }^{17} \\
(2010) \\
1-\end{array}$ & $\begin{array}{l}\mathrm{n}=56 \text { (28 de } \\
\text { cada grupo) } \\
\text { Reino Unido. }\end{array}$ & ECA & 6 meses. & $\begin{array}{l}\text { Comparar el dolor a } \\
\text { la punción. }\end{array}$ & $\begin{array}{c}\text { Los pacientes y el personal prefieren la } \\
\text { punción del ojal, reduce las molestias } \\
\text { durante la punción. }\end{array}$ \\
\hline $\begin{array}{l}\text { Chow }^{18} \\
(2011) \\
1-\end{array}$ & $\begin{array}{c}\mathrm{n}=70 \text { (35 } \\
\text { técnica } \\
\text { ojal vs } 35 \\
\text { convencional) } \\
\text { Australia. }\end{array}$ & & & $\begin{array}{l}\text { Múltiples resultados } \\
\text { relacionados con las } \\
\text { complicaciones. }\end{array}$ & $\begin{array}{l}\text { La técnica del ojal produjo más infecciones, } \\
\text { formación de hematomas y dolor en el } \\
\text { sitio durante la diálisis que con el grupo de } \\
\text { rotación de la escalera de cuerda. }\end{array}$ \\
\hline $\begin{array}{l}\text { MacRae }^{19} \\
(2012) \\
1+\end{array}$ & $\begin{array}{l}\text { n=140 (70 por } \\
\text { cada grupo }) \\
\text { Canadá. }\end{array}$ & ECA & 12 meses. & $\begin{array}{l}\text { Múltiples resultados } \\
\text { relacionados con las } \\
\text { complicaciones. }\end{array}$ & $\begin{array}{l}\text { A las } 8 \text { semanas el dolor fue similar en } \\
\text { ambos grupos. }\end{array}$ \\
\hline $\begin{array}{l}\text { Guixian } \text { Chen }^{20} \\
\text { (2012) } \\
1-\end{array}$ & $\begin{array}{l}\mathrm{N}=40 \\
\text { China. }\end{array}$ & ECA & 12 meses. & $\begin{array}{l}\text { Múltiples resultados } \\
\text { relacionados con las } \\
\text { complicaciones. }\end{array}$ & No hubo diferencias significativas. \\
\hline $\begin{array}{l}\text { Vaux }{ }^{21} \\
(2013) \\
1-\end{array}$ & $\begin{array}{c}\mathrm{N}=127 \text { (58 } \\
\text { técnica } \\
\text { ojal vs } 69 \\
\text { convencional) } \\
\text { Reino Unido. }\end{array}$ & ECA & 12 meses. & $\begin{array}{l}\text { Múltiples resultados } \\
\text { relacionados con las } \\
\text { complicaciones. }\end{array}$ & $\begin{array}{c}\text { No hubo diferencias significativas en los } \\
\text { tiempos de sangrado y el uso de lidocaína } \\
\text { entre los } 2 \text { grupos. }\end{array}$ \\
\hline $\begin{array}{l}\text { Grudzinski22 } \\
\text { (2013) } \\
1-\end{array}$ & $\begin{array}{l}23 \text { Estudios } \\
\text { Canadá. }\end{array}$ & $\begin{array}{l}\text { Rev. } \\
\text { Sistemá- } \\
\text { tica. }\end{array}$ & $\begin{array}{l}\text { Entre } 3 \text { y } 18 \\
\text { meses. }\end{array}$ & $\begin{array}{l}\text { Múltiples resultados } \\
\text { relacionados con las } \\
\text { complicaciones. }\end{array}$ & $\begin{array}{c}\text { Concluyen que la técnica del ojal necesita } \\
\text { estudios más amplios y definitivos para } \\
\text { determinar si esta es segura para un uso } \\
\text { más amplio. }\end{array}$ \\
\hline $\begin{array}{l}\text { Wenyan }{ }^{23} \\
\text { Qian (2014) } \\
\text { 1- }\end{array}$ & $\begin{array}{l}\mathrm{N}=70 \text { (40 } \\
\text { para el grupo } \\
\text { experimental, } \\
30 \text { al grupo } \\
\text { control) } \\
\text { China. }\end{array}$ & ECA & 12 meses. & $\begin{array}{l}\text { Múltiples resultados } \\
\text { relacionados con las } \\
\text { complicaciones. }\end{array}$ & No hubo diferencias significativas. \\
\hline $\begin{array}{l}\text { Wong }{ }^{24} \\
(2014) \\
1-\end{array}$ & $\begin{array}{l}23 \text { Estudios } \\
\text { Canadá. }\end{array}$ & $\begin{array}{l}\text { Rev. Sis- } \\
\text { temática. }\end{array}$ & $\begin{array}{l}\text { Entre } 3 \text { y } 18 \\
\text { meses. }\end{array}$ & $\begin{array}{l}\text { Múltiples resultados } \\
\text { relacionados con las } \\
\text { complicaciones. }\end{array}$ & $\begin{array}{l}\text { Hubo diferencia entre en dolor en las } \\
\text { técnicas en estudios observacionales, sin } \\
\text { embargo en ECA estas no eran evidentes. }\end{array}$ \\
\hline $\begin{array}{l}\text { Chong } 25 \\
(2016) \\
1++\end{array}$ & $\begin{array}{l}n=861 \\
\text { China. }\end{array}$ & $\begin{array}{c}\text { Metaanálisis } \\
8 \text { ensayos } \\
\text { controlados } \\
\text { aleatorios } \\
\text { y } 2 \text { ensayos } \\
\text { controlados } \\
\text { cuasialeato- } \\
\text { rios. }\end{array}$ & $\begin{array}{l}\text { Entre 6-12 } \\
\text { meses. }\end{array}$ & $\begin{array}{l}\text { Múltiples resultados } \\
\text { relacionados con las } \\
\text { complicaciones. }\end{array}$ & $\begin{array}{l}\text { Al comparar las dos técnicas no hubo } \\
\text { reducción al dolor. }\end{array}$ \\
\hline
\end{tabular}

Nota: ECA: Ensayo controlado aleatorio; Nivel de evidencia 1-: ECA con riesgo alto de sesgo. 1+: ECA con riesgo bajo de sesgo. 1++: ECA con riesgo muy bajo de sesgo. 
El tamaño muestral está constituida por 3455 pacientes. Disgregando la muestra en función de la tipología de estudio, se obtiene que la mayoría están representados por los estudios de revisión sistemática $n=2952$, respecto a los 503 pacientes que se utilizaron para los ensayos clíni$\cos (n=250$ grupo experimental y $n=253$ grupo control).

La totalidad de los ensayos abordan el dolor a la canalización ${ }^{17-25}$, no obstante, no es la única variable objeto de estudio. Ocho de los 9 artículos valoran la infección tras canalización ${ }^{17-23,25}$. Respecto a la infección, los datos no fueron concluyentes ya que existían diferencias entre las definiciones de infección y metodología de estudios.

Siete de los 9 estudios valoran la prevalencia de aneurisma en función de la técnica de abordaje. Se concluye que la técnica de ojal tiene menor prevalencia de aneurisma en relación a la técnica de escalera ${ }^{17,} 20-25$.

Respecto a la formación de hematomas, se valora en el $77 \%$ de los estudios consultados. Al igual que la variable infección, hubo diferencia entre la definición de ésta y la metodología de estudio 17-20,22,24,25.

En la misma línea, se valora el tiempo de sangrado post-canalización. Las diferencias metodológicas entre los artículos dificultan llegar a un consenso, sin embargo, no se notifica un mayor sangrado en la técnica del ojal.

En relación a la aparición de trombosis o estenosis tras la canalización ${ }^{17,20,23}$ se concluye que la técnica del ojal tiene una tasa significativamente inferior de estenosis respecto a la técnica de escalera.

Finalmente, Vaux et al., ${ }^{21}$ abordan el número de intervenciones y la supervivencia de la fistula comparando la técnica de ojal respecto a las técnicas convencionales. Los resultados muestran un menor número de intervenciones en la técnica del ojal y una mayor tasa de supervivencia. No obstante, en el mismo estudio se reconoce un sesgo respecto a este hallazgo, ya que el grupo de técnica del ojal parte de un menor número de accesos previos respecto al grupo convencional.

En el metaanálisis se muestran los resultados relacionados con el dolor entre las dos técnicas (ver Figura 2).

\section{Discusión}

Respecto a las distintas técnicas de punción, no existe consenso en la literatura científica para determinar

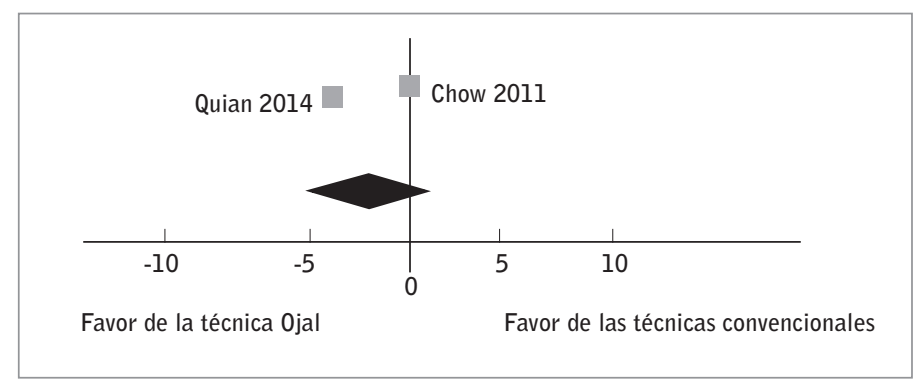

Figura 2. Diagrama Forest plot.

qué técnica produce menos dolor al paciente. Los resultados de los ensayos clínicos consultados indican que no se produce una reducción significativa del dolor durante la punción del FAV con el uso de la técnica del ojal frente a las técnicas tradicionales ${ }^{17-21,23,25}$. Esta falta de consenso se hace más patente en las revisiones sistemáticas realizadas por Wong et al., y Grudzinski et al,.22,24 En ellas se puede observar como algunos estudios de corte descriptivo tienden a dar un mayor beneficio contra el dolor a la técnica del ojal. Sin embargo cuando se valoran ensayos clínicos las diferencias no son suficientes para determinar que técnica produce un menor dolor en el paciente.

Entrando de forma más específica en materia, se puede poner de ejemplos los estudios realizados por Struthers et al., MacRae et al., y Chow et al., donde se valoró de forma específica el dolor comparando el método del ojal frente a la canalización en escalera. En todos estos estudios no se encontraron diferencias significativas entre ambas técnicas respecto al dolor $^{17-19}$. En esa misma línea está el estudio realizado por Vaux et al. que tampoco evidencia diferencias importantes en la canalización mediante las dos técnicas expuestas. Es más, 8 pacientes ( $11 \%$ de la muestra) no toleraron la técnica del ojal y tuvieron que pasarse a técnicas tradicionales ${ }^{21}$.

Finalmente, si valoramos el metaanálisis realizado por Chong et al en 2016 que recoge 8 ECA desde el año 2003 hasta 2014. Se valoraron las complicaciones en función del método de acceso venoso. Tras el pertinente estudio estadístico mediante diferentes pruebas se determinó que el dolor muestra resultados contradictorios y por tanto el metaanálisis solo puede ser usado como referencia en este campo (DME $=-1,48$, IC 95\% $(-4,41,1,18), P>0,05)^{25}$.

En general, la calidad de los estudios fue media según la escala CASPe (un estudio categoría $\mathrm{A}$ y el resto ca- 
tegoría B según el manual Cochrane para Revisiones Sistemáticas de Intervenciones (Versión 5.1.0, 2011). No incluir estudios de carácter descriptivo da mayor potencia a la revisión sistemática. Sin embargo, la mayoría de los ECA carecían de cegamiento, ocultación de la asignación, bajo tamaño muestral y la evaluación del dolor se realizaba con diferentes herramientas. Además, las dos revisiones sistemáticas incluidas presentan sesgo de selección al incluir estudios de diferente calidad metodológica. Otro hándicap presente es la duración del seguimiento que varió de 6 a 12 meses y la heterogeneidad de los resultados del estudio ya que en la mayoría valoraban otras variables diferentes al dolor. Además, solo identificamos estudios publicados en inglés y español, por lo que es posible que no se cubran todos los estudios.

Después de revisar la literatura científica y analizar los resultados obtenidos, se extrae la siguiente conclusión: el grado de dolor que se genera durante la punción de la fístula arteriovenosa no difiere entre las distintas técnicas de punción empleadas, ya sea la técnica del ojal, o bien, la técnica punción en escalera o la técnica área de punción.

En relación con futuras líneas de investigación, sería conveniente realizar estudios experimentales de mayor envergadura donde se compare el método ojal con el método "área de punción", ya que es la técnica más utilizada a nivel nacional. Además, los estudios que se realicen en un futuro deberían tener un periodo de seguimiento más largo, contar con una muestra mayor y homogenizar las herramientas para medir el dolor.

\section{Declaración de autores}

Los autores declaran no tener conflicto de intereses.

Recibido: 30 septiembre 2018

Revisado: 2 noviembre 2018

Modificado: 6 febrero 2019

Aceptado: 10 febrero 2019

\section{Bibliografía}

1. López-González A, Fernández-Rivera A, Díaz-Rodriguez L, Ornosa-Agra C. Impacto del calibre de las agujas en la calidad de la hemodiálisis. Enferm. Nefrol. 2015;18(2):118-22.

2. Fernández-Castillo $R$, Cañadas de la Fuente $G A$, Fernández-Gallegos R, Cañadas de la Fuente GR. Efecto de la posición del bisel de la aguja en el acceso a la fístula arteriovenosa interna. Rev Soc Esp Enferm Nefrol. 2008;11(4):254-8.

3. Barba-Velez A, Ocharán-Corcuera J. Manejo de los accesos vasculares para hemodiálisis. Gac Med Bilbao. 2011;108(4):108-13.

4. Grau-Pueyo C, Granados-Navarrete I, Moya-Mejía C, García-Blanco M, García-Ciaño XV, Ramírez-Vaca J, et al. La punción del acceso vascular en hemodiálisis es una necesidad, el método Buttonhole una opción. Rev Soc Esp Enferm Nefrol. 2011;14(1):30-6.

5. Moreno-Núñez E, Arenas-Jiménez MD, Porta-BeIlmar E, Escalant-Calpena L, Cantó-García MJ, Castell-García $G$, et al. Estudio de la prevalencia de trastornos ansiosos y depresivos en pacientes en hemodiálisis. Rev Soc Esp Enferm Nefrol. 2004;7(4):225-33.

6. Baena-Ruíz L, Martín-González B, Marcos-Ayuso A. Implantación de la técnica del "ojal" o "buttonhole" en una unidad de hemodiálisis hospitalaria: satisfacción del paciente. Enferm. Nefrol. 2015;18(1):61-4.

7. Granados-Navarrete I, Abril-Sabater D, Alcaraz-Busqueta $\mathrm{F}$, Mañé-Buixo $\mathrm{N}$, Padilla-Ruiz J, Real-Gatius J. Una actuación de enfermería: intentar aliviar el dolor en las punciones de hemodiálisis. Rev Soc Esp Enferm Nefrol. 2005;8(3):231-6.

8. González-Molina J, Momblanch-Amoros T, Moreno-Aliaga C, Sanz-Escriba A, Navarro Daudén L, Martí i Monros A, et al. Análisis enfermero de la presencia de la Técnica del 0jal en las unidades de hemodiálisis españolas. Enferm Nefrol. 2013;16(2):93-8.

9. Twardowski Z, Kubara H. Different sites versus constant sites of needle insertion into arteriove- 
nous fistulas for treatment by repeated dialysis. Dial Transplant. 1979;8(10):978-80.

10. Krönung G. Plastic deformation of Cimino fistula by repeated puncture. Dial Transplant. 1984;13:635-8.

11. Kosa SD, Bhola C, Lok CE. Hemodialysis patients' satisfaction and perspectives on complications associated with vascular access related interventions: are we listening?. J Vasc Access. 2016;12; 17(4):313-9.

12. Urrutia G, Bonfill X. Declaración PRISMA: una propuesta para mejorar la publicación de revisiones sistemáticas y metaanálisis. Med Clin (Barc) 2010; 135:507-11.

13. Cué $M$, Díaz G, Díaz AG, Valdés MC. El artículo de revisión. RESUMED. 1996;9(2):86-96.

14. Cabello JB. Plantilla para ayudarte a entender un Estudio de Ensayo clínico. En: CASPe. Guías CASPe de Lectura Crítica de la Literatura Médica. Alicante: CASPe; 2005. Cuaderno I: 5-8.

15. Higgins JPT, Green S. Manual Cochrane de Revisiones Sistemáticas de Intervenciones, versión 5.1.0. The Cochrane Collaboration [Internet]. 2011. [Consultado 18 Feb 2018]. Disponible en: https:// es.cochrane.org/sites/es.cochrane.org/files/public/ uploads/Manual_Cochrane_510_reduit.pdf

16. Moher D, Liberati A, Tetzlaff J, Altman DG. The PRISMA Group. Preferred Reporting Items for Systematic Reviews and Meta-Analyses: The PRISMA Statement. PLoS Med. 2009 6(6): e1000097.

17. Struthers J, Allan A, Peel RK, Lambie SH. Buttonhole needling of ateriovenous fistulae: a randomized controlled trial. ASAIO J. 2010; 56(4):319-22.
18. Chow J, Rayment G, San Miguel S, Gilbert M. A randomised controlled trial of buttonhole cannulation for the prevention of fistula access complications. $J$ Ren Care. 2011;37(2):85-93.

19. MacRae JM, Ahmed SB, Atkar R, Hemmelgarn BR. A randomized trial comparing buttonhole with rope ladder needling in conventional hemodialysis patients. Clin J Am Soc Nephrol. 2012; 7(10): 1632-8.

20. Chen G, Xiao H, Yang Z. Application of tunnel fistula puncture in patients with maintenance hemodialysis. J Nurs. 2012;19:48-9.

21. Vaux E, King J, Lloyd S, Moore J, Bailey L, Reading I, et al. Effect of buttonhole cannulation with a polycarbonate PEG on in-center hemodialysis fistula outcomes: a randomized controlled trial. $A m \mathrm{~J}$ Kidney Dis. 2013;62(1):81-8.

22. Grudzinski A,Mendelssohn D,Pierratos A,Nesrallah G. A Systematic Review of Buttonhole Cannulation Practices and Outcomes. Semin Dial. 2013;26(4): 465-75.

23. Qian W, Yu J, Zheng J. The Reduction of Arteriovenous Fistula Complications of Blunt Needle Buttonhole Methods. Nurs Reha J 2014;13:1073-4.

24. Wong $B$, Muneer $M$, Wiebe $N$, Storie $D$, Shurraw $\mathrm{S}$, Pannu N, et al. Buttonhole Versus Rope-Ladder Cannulation of Arteriovenous Fistulas for Hemodialysis: A Systematic Review. Am J Kidney Dis. 2014;64(6):918-36.

25. Chong Ren, Xin Han, Bihong Huang, Li Yuan, Yanpei Cao, Xiaoli Yang. Efficacy of buttonhole cannulation $\mathrm{BH})$ in hemodialysis patients with arteriovenous fistula: a meta-analysis. Int J Clin Exp Med 2016; 9(8):15363-70.

Este artículo se distribuye bajo una Licencia Creative Commons Atribución-NoComercial 4.0 Internacional. https://creativecommons.org/licenses/by-nc/4.0/

Open Access (C) (1) (8) 